\title{
The Combination of Levomilnacipran, Cognitive Behavior Therapy and Christian Based Self - Help Group in the Treatment of Bulimia Nervosa: A Case Report
}

Hani Raoul Khouzam*

Monterey County Department of Health, Behavioral Health Bureau, Salinas, CA

\begin{abstract}
Bulimia nervosa is a severe, life-threatening feeding and eating disorder. This report describe a case of 33 year old female with chronic bulimia nervosa , who favorably responded to the combination of a Cognitive behavior therapy and Christian based self -help group and the serotonin norepinephrine reuptake inhibitor antidepressant levomilnacipran which has only been approved for the treatment of major depressive disorder. Controlled trials would need to be conducted to confirm the effectiveness of this combination in the treatment of other patients with bulimia nervosa.
\end{abstract}

Keywords: Eating disorders; Bulimia nervosa; Pharmacology; Christian self-help groups

\section{Introduction}

Bulimia nervosa (BN) is a feeding and eating disorder which is characterized by frequent episodes of binge eating followed by inappropriate behaviors such as self-induced vomiting, misuse of laxatives, diuretics, or other medications in addition to fasting, or excessive exercise to avoid weight gain [1]. Patients with BN usually experience a sense of lack of control over their excessive eating and some feel guilty and depressed due to their uncontrolled eating habits $[2,3]$. Although chronic $\mathrm{BN}$ is considered a severe and life-threatening condition that could be difficult to detect since many patients maintain a normal body weight, it can be safely when it is accurately identified $[2,3]$. The treatment of $\mathrm{BN}$ usually combine psychotherapy and pharmacology [3]. Although many different psychotherapy types have been used for the treatment $\mathrm{BN}$, cognitive behavioral therapy (CBT) has been the most successful $[4,5]$. The selective serotonin reuptake inhibitors (SSRIs) are the psychopharmacological agents that have been most used in the treatment of $\mathrm{BN}$ [6]. Among the SSRIs fluoxetine is the only pharmacological agent that has been approved in the US by the Food and Drug Administration (FDA) for the treatment of BN [7]. Fluoxetine (Prozac ${ }^{\circ}$ ) has shown beneficial clinical effects in decreasing the binging and purging cycle, and in preventing relapse [8]. Clinicians have used other agents beside the SSRIs, including serotonin norepinephrine reuptake inhibitors (SNRIs), various tricyclic antidepressants (TCAs), mirtazapine, antiepileptic such as topiramate and the antiemetic ondansetron [6-8]. Despite its possible effectiveness, the antidepressant bupropion is contraindicated because of the association of its use with seizures in patients who purge and its anorexic side effects [9]. Medications that require dietary restrictions, such as the monoamine-oxidase inhibitors (MAOIs) are less appropriate in $\mathrm{BN}$ due to the medical complications associated with the diet restrictions nonadherence [10].

This report describe a case of 33 year old female with chronic BN, who favorably responded to the SNRI antidepressant Levomilnacipran which is only approved for the treatment of Major Depressive Disorder (MDD )in combination with CBT and a Christian based self -help group. To the author knowledge there has been no published studies examining the effects of Levomilnacipran or Christian based self-help group on BN treatment, controlled trials for this indication would be useful.

\section{Case Report}

Ms. $\mathrm{R}$ was a 33-year-old female, who developed $\mathrm{BN}$, at the age of 17. Her eating episodes usually began following each dinner meals. She would ask her parents to be excused to complete her homework and instead she will consume large quantity of food that she stored in a big ice cooler and hidden in her closet under several items of clothing. She would usually eat 2 medium size boxes of chocolate cookies, a large bag of potato ships, a medium size box of cheese crackers, 3 bowls of cereal with 2 cups of chocolate milk, and one bag of $M$ and $\mathrm{M}$ candies. Following each episode of food consumption she would immediately self-induce emesis. Because she used most of her weekly allowance and the extra money she earned from babysitting in food purchases, her parents became suspicious about possibility of alcohol or drug use, however one of her close friends who accidently discovered the ice cooler informed her parents. The parents understood their daughter's eating struggles since her maternal aunt had suffered from binge eating during her adolescence. Based on the school psychologist recommendation Ms. R was referred for CBT and received one hour weekly session over a 12 weeks period. The CBT for BN included the following elements: commitment to change, self-monitoring, healthy eating pattern, food avoidance, weekly weighing, patient education, cues and consequences, chains of behaviors, stress management and problem-solving skills, cognitive restructuring, body image, and assertiveness. Ms. R successfully completed the 12 weeks of CBT and as a result experienced a remission of $\mathrm{BN}$.

At the age of $21 \mathrm{Ms}$. R's BN escalated to nightly episodes of consuming 3 peanut jelly sandwiches, 2 cartons of chocolate milk, a

*Corresponding author: Hani Raoul Khouzam, Monterey County Department of Health, Behavioral Health Bureau, Salinas, CA, 1441 Constitution Blvd, Building 400, Suite 202.Salinas, CA93906, Tel: 559930 6406; E-mail: khouzamh@ co.monterey.ca.us or hrmdkhouzam@gmail.com

Received: October 14, 2016; Accepted: November 16, 2016; Published: November 23, 2016

Citation: Khouzam HR (2016) The Combination of Levomilnacipran, Cognitive Behavior Therapy and Christian Based Self -Help Group in the Treatment of Bulimia Nervosa: A Case Report. Clin Exp Psychol 2: 142. doi: 10.4172/24712701.1000142

Copyright: (c) 2016 Khouzam HR. This is an open-access article distributed under the terms of the Creative Commons Attribution License, which permits unrestricted use, distribution, and reproduction in any medium, provided the original author and source are credited. 
box of cheese crackers, a tub of tapioca rice pudding and a medium size jar of strawberry jam followed by purging. At the time she was working as an $8^{\text {th }}$ grade teacher assistant and had many overweight children in her classroom, she was extremely frightened by the thought of not being able to control her weight. She began to use laxatives, she also joined a physical fitness club and exercised on a daily basis. Despite the purging, the exercise, the use of laxatives and the maintenance of an ideal body weight, she was overwhelmed and preoccupied with constant rumination of seeing herself as an overinflated balloon.

Ms. R did not use alcohol or illicit drugs; she did not smoke cigarettes or drink any caffeinated beverages. Her parents encouraged her to seek professional help. She was evaluated by her family physician. She had a normal physical examination, and did not have any co-occurring medical or psychiatric conditions and was not taking any prescribed or over the counter mediations. She was referred to a psychiatrist and was treated with several SSRIs, beginning with fluoxetine, and then changed to sertraline, then paroxetine, and lastly citalopram. Although these medications where given at therapeutic doses, she could not tolerate their side effects of headaches, dizziness and constipation. She was disappointed with the lack of progress and stopped seeing the psychiatrist and continued to purge, to use laxative and to avidly exercise and kept an ideal body weight. Ms. R was described as an attractive and bright young lady who was also kind and very well-liked by her friends. Although she possessed a unique style of personal, social and occupational functioning. She did not date. She did not want to be married or to have children until she overcame her struggle with BN. Although she never expressed feelings or intention of self-harm, she described feelings of desperation and earnestly wanted to be "cured" from $\mathrm{BN}$.

Based on her past history of success with CBT for BN and her intolerance to SSRIs, Ms. R agreed to be treated with the combination of CBT with a non SSRI's medication. Treatment was initiated with the SNRI venlafaxine. Despite a gradual titration of venlafaxine to $150 \mathrm{mg} /$ day and continued weekly CBT psychotherapy, no improvement was noted over the course of 6 weeks. She also developed a new onset of hypertension which was attributed to venlafaxine because her blood pressure normalized when it was discontinued.

She expressed feelings of sadness and worries without the emergence of suicidal ideation or intention and did not want to maintain CBT due to conflicts with her new job as a special education teacher.

She agreed to consider another SNRI Duloxetine which was initiated at the dose of $20 \mathrm{mg} /$ day however she complained of jitteriness and headaches and did not want to maintain duloxetine.

Ms. R became increasingly distraught and concerned and was about to quit treatment altogether. Her parents intervened and she agreed to give medication a last chance. She consented to take the newly approved SNRI antidepressant Levomilnacipran which was Initiated at the dose of $20 \mathrm{mg} /$ day for 2 days, then increased to $40 \mathrm{mg} /$ day which she tolerated well without adverse effects. Within a four weeks period, the number of binging and induced emesis decreased to 4 times a week, however the drive to binge was still strong despite her persistent efforts to avoid all food related triggers.

She agreed to resume weekly CBT sessions. Despite the noticeable CBT beneficial effects on markedly decreasing the urges to overeat, Ms. $\mathrm{R}$ relapsed and returned back to her former pattern of daily overeating and inducing emesis. She expressed overwhelming feelings of hopelessness, helplessness with the emergence for the first time of suicidal intention with a plan to jump over a bridge into an iced frozen ravine near her house.
She agreed to be voluntarily admitted to the inpatient psychiatry unit. During her first hospital day she consented to an increase of Levomilnacipran to $80 \mathrm{mg} /$ day which she tolerated well. Although the urges to overeat and the thought of inducing emesis subsided for five consecutive days. Ms. $\mathrm{R}$ attributed these beneficial effects to the strictly supervised hospital milieu and did not believe that her BN was well controlled.She also a continued to verbalize intention to jump over the bridge when she is discharged.

On day seven another female patient who was admitted to the hospital due to self-injurious behaviors was visited by her roommate who happened to know Ms. R because they both attended the some middle school and as they began to catch-up with their life's journeys the friend reported that she had also suffered from BN. She also mentioned that she was healed from this affliction by attending a Christian selfhelp group.

On day ten of inpatient hospitalization the suicidal intention along with the urge to overeat and to induce emesis subsided and Ms. R was discharged to outpatient follow-up and since she expressed an interest in attending the BN Christian self-help group, her discharge plan coordinator inquired about its location and found it in a nearby town.

Ms. R resumed her employment as special education teacher, and continued Levomilnacipran treatment at the dose of $80 \mathrm{mg} /$ day. She did not feel the need to attend further CBT Sessions and was confident about her gained skills in practicing its techniques in resisting the urges to overeat. At this point of time, she experienced a high level of optimism about her $\mathrm{BN}$ recovery and attributed her progress to practicing CBT techniques combined with Levomilnacipran treatment. Despite her initial interests in attending BN Christian self-help group, she did not feel that she needed this intervention.

For nearly 4 months she remained in $\mathrm{BN}$ remission until she met her colleague, a new male employee who expressed interests in dating. Since she was confident about her recovery and ongoing progress she accepted his offer. However he was eager to formalize their relationship and they became engaged. Two months later while visiting her fiancés parents and without any precipitating stressors the drive and urges to overeat intensified. Ms. $\mathrm{R}$ was unable to create any excuses that would allow her to leave the dinner table so she could purchase food from a supermarket that was about 20 miles away.

Her fiance's parents perceived her marked feelings of distress as an expression of needing to have some personal time with their son. They graciously offered them the opportunity to go for a walk.

Mr. $\mathrm{R}$ confined her concerns and fears about relapsing into uncontrolled BN and felt unworthy of marrying or of having a family. With her fiancés support she sought an urgent appointment with her psychiatrist who recommended an increase in Levomilnacipran dose from $80 \mathrm{mg}$ to $120 \mathrm{mg} /$ day. She was reluctant to follow through with this suggestion and asked if she could rethink it over. She then remembered her high school classmate conversation related to the $\mathrm{BN}$ Christian self-help group. She attended the group on a weekly basis and was able to resist the urges to overeat. Her fiancé who described himself as an agnostic was very skeptical about such an intervention so he was invited to attend a special session for family and significant others. The BN Christian support group was based on the premises that people who are inflicted with an eating disorder in general have a negative image and perceptions of God because they fear abandonment by God due to their dishonest and deceptive patterns of food intake. They also have difficulty surrendering their will to a loving and forgiving God. As a result they are constantly overwhelmed by feelings 
of spiritual unworthiness, shame and guilt. In order to change these patterns the group members become accountable to each other in reporting their episodes of overeating and inducing emesis and rather than feeling guilty and unworthy, they would ask to be forgiven and to be unconditionally accepted and supported in seeking God's guidance to resist the urges to overeat. Despite her initial doubts, Ms. R agreed to practice these newly taught spiritual concepts and was unbelievably surprised by their positive effects on strengthening her personal faith and in helping her resist all the temptations to overeat. She described these Christian concepts as, life changing, uplifting and healing. She particularly attributed her BN recovery to memorizing a special Bible verse Proverbs 3:5-6 "Trust in the Lord with all your heart, and do not lean on your own understanding. In all your ways acknowledge him, and he will direct your paths". In addition the Christian self -help group included the option of incorporating personal prayers that empathized the concepts "save," "heal," "preserve," and "rescue" as they related to emotional wellbeing through "inner healing".

Over the course of nine months, Ms. R continued to attend the weekly BN Christian self -help group in addition to practicing CBT techniques and taking Levomilnacipran at the dose $120 \mathrm{mg} /$ day. During this time, she continued to excel in her job as a special education teacher and she and her fiancé are preparing for their wedding which is set for an upcoming Valentine day.

\section{Discussion}

According to the American Psychiatric Association Practice Guidelines for Eating Disorders [11], the Initial care for BN is usually provided in outpatient settings. Hospitalization is recommended in cases of medical complications due significant metabolic abnormalities, increased suicide risk, medical complications, inability to care for self and lack of outpatient treatment response [12]. Ms. R did not develop medical complications and was always able to care for herself, but was hospitalized when she was discouraged about her failed outpatient treatment and expressed suicidal intention.

Among the many types of psychotherapy, CBT has been proven through evidence-based practices, to be an effective treatment for $\mathrm{BN}$ $[4,5,13]$. Ms. $\mathrm{R}$ gained skills in practicing various CBT techniques to avoid and counteract undesirable over eating habits. CBT approaches also were essential in helping her identify, examine, and address, her dysfunctional thoughts, and urges related to overeating, then inducing emesis [13]. Despite the favorable response, CBT alone was not sufficient without the combination of pharmacological intervention [13].

Antidepressants are considered the main pharmacological agents that are used in the treatment of $\mathrm{BN}[2,6]$. They are also beneficial in treating concurrent conditions especially depression, anxiety, obsessions, and certain impulse disorders [6]. Among the antidepressants, the SSRIs have been the most effective, although their exact mechanism in treating BN is still unknown, it is presumed to be mediated through their effects on the brain serotonin systems $[12,14]$. Among the SSRIs, fluoxetine is the only one that is approved by the FDA for the treatment of BN [7]. Ms. R could not tolerate fluoxetine side effects and the other SSRIs including sertraline, paroxetine, and lastly citalopram. Among the other antidepressants bupropion is considered relatively contraindicated in $\mathrm{BN}$ treatment due to its risk of inducing seizures especially in patients with eating disorders $[9,12]$. The TCAs desipramine, amitriptyline and imipramine have also been useful in some patients with $\mathrm{BN}$ but due to their high risks of adverse effects including possible excessive weight gain and lethality in overdose they have not been recommended as initial pharmacological interventions
$[6,7,12]$. The MAOIs antidepressants such as phenelzine which seemed to be beneficial in $\mathrm{BN}$ treatment its required dietary restrictions and its interactions with a wide variety of non-prescription medications usually used by BN patients make this class less appropriate for treatment of this condition [10]. Other antidepressant such as trazodone and mirtazapine, and mood stabilizers such as, topiramate, lithium, valproic acid have also been used with some effectiveness in BN [6-8,12-14]. Other agents including odansetron, d-fenfluramine, baclofen, orlistat, antiandrogenic oral contraceptive, human growth hormone, cannabis, zinc, clonidine, antihistaminics, prokinetic agents, methylphenidate, the atypical or second generation antipsychotics such as olanzapine and aripipriazole, have been also used as alternative options in the management of $\mathrm{BN}$ all with limited beneficial effects [12-18].

Several case reports suggest that the SNRIs such as venlafaxine [19] and duloxetine [20] through their dual serotonin and norepinephrine reuptake inhibitory effects can produce a positive, and synergistic effect in the treatment of BN. Because Ms. R developed unfavorable side effects to venlafaxine, and duloxetine, it seemed clinically appropriate to consider the novel SNRI Levomilnacipran as a viable treatment option and it fortunately resulted in a noticeable improvement, however to this author knowledge there have been no other prospective double blind placebo control studies that have conducted to confirm such an effect on other patients with BN.

Clinicians prescribing Levomilnacipran need to be aware of its potential adverse effects [21], as with all antidepressants, it carries a warning of increased risk of suicidal thoughts and behavior in patients 24 years or younger, and it has not been studied in patients younger than 18 years [22]. Levomilnacipran increases systolic and diastolic blood pressure by a median of $4 \mathrm{mmHg}$ and $2.5 \mathrm{mmHg}$, respectively [21], and $3 \%$ of patients (95\% confidence interval, $1 \%$ to $6 \%$ ) with normal blood pressure or prehypertension will develop hypertension[23]. It is advisable not to use it in patients with poorly controlled hypertension [22]. Although, it does not cause clinically significant changes in basic laboratory tests or QT prolongation [21,23-26]. Levomilnacipran should not be used with other serotonergic agents, such as MAOIs, triptans, tramadol, and other antidepressants to minimize the risk of serotonin syndrome [22]. The dosage of levomilnacipran should be limited to $80 \mathrm{mg}$ per day when used concurrently with a cytochrome P450 3A4 inhibitor because levomilnacipran is partially metabolized by the liver, and medications that inhibit the cytochrome P450 3A4 system, such as ketoconazole, may cause it to accumulate [22]. Patients with moderate and severe renal impairment should be advised to consider a lower dose.

Self -help support groups and 12-step programs such as Overeaters Anonymous may be helpful as adjuncts in initial treatment and for subsequent relapse prevention in BN. To determine the efficacy of these groups; a Cochrane Database Systematic Review was conducted and concluded with mixed results about their effectiveness [27]. Another randomized, control group design study to evaluate the effectiveness of a spiritual group intervention for inpatient eating disorders compared the effectiveness of a Spirituality group with Cognitive and Emotional Support groups [28]. On weekly outcome measures, patients in the Spirituality group improved significantly more quickly during the first four weeks of treatment, thus suggesting preliminary evidence that promoting spiritual growth and well-being of patients with eating disorders patients could contribute to a reduction in their symptoms [28]. In another systematic study that examined the effects of religiosity, spirituality on eating disorders and body image concerns recommended pursuing further investigation into the protective benefits of religiosity and spirituality in the progression of eating disorders [29]. Although 
Citation: Khouzam HR (2016) The Combination of Levomilnacipran, Cognitive Behavior Therapy and Christian Based Self -Help Group in the Treatment of Bulimia Nervosa: A Case Report. Clin Exp Psychol 2: 142. doi: 10.4172/2471-2701.1000142

Page 4 of 4

there is an overlap in some components of CBT and Christian based self -help group. Compared to Christian based self -help group which are based on each individual faith and prayer patterns, CBT have the advantage of its structured format which can be generalized to all the population regardless of their personal faith. CBT IN addition have a wider availability of its formal guidelines and recommendations. Ms. R's favorable response to the Christian self-help group seems to parallel this conclusion; however it cannot be generalized to other BN cases without further prospective double blind placebo control studies.

\section{Conclusion}

Several placebo-controlled studies investigating the use of SSRIs in the treatment of $\mathrm{BN}$ underscore some efficacy in their use with fluoxetine being the only medication of that class which has been FDA approved for $\mathrm{BN}$ treatment. Moreover, several case reports suggest that the SNRIs can also reduce BN. Assuming that both the serotonin and norepinephrine systems are implicated in the pathophysiology of BN, it is reasonable to believe dual serotonin and norepinephrine reuptake inhibitor agents, such as venlafaxine and duloxetine could produce a positive, and synergistic effect in the treatment of this debilitating eating disorder. Because Ms. $\mathrm{R}$ developed unfavorable side effects to venlafaxine, and duloxetine, it seemed clinically appropriate to consider the novel SNRI levomilnacipran as a viable treatment option, and it fortunately resulted in a noticeable improvement. This case report also provided additional support for the combination of levomilnacipran, CBT and Christian-based self-help group as an effective intervention in the treatment refractory $\mathrm{BN}$ and highlights the need for further research to confirm these positive outcomes in other patients who suffer from this debilitating illness.

\section{Acknowledgments}

The author thank Dr. David P Soskin and Dr. Oriana P Vesga-Lopez for their support and Dr. Avak A Howsepian, Dr. William C Torrey, Dr. Alan I Green and Dr. Heba Gad for their encouragement.

\section{References}

1. Herpertz-Dahlmann B (2015) Adolescent eating disorders: update on definitions, symptomatology, epidemiology, and comorbidity. Child Adolesc Psychiatr Clin 24: 177-196.

2. Keel PK, Brown TA (2010) Update on course and outcome in eating disorders. Int J Eat Disord 43: 195-204.

3. Smink FR, van Hoeken D, Hoek HW (2013) Epidemiology, course, and outcome of eating disorders. Curr Opin Psychiatry 26: 543-548.

4. Waller G, Gray E, Hinrichsen H, Mountford V, Lawson R, et al. (2014) Cognitive-behavioral therapy for bulimia nervosa and atypical bulimic nervosa: effectiveness in clinical settings. Int J Eat Disord 47: 13-17.

5. Thompson-Brenner H, Shingleton RM, Sauer-Zavala S, Richards LK, Pratt EM (2015) Multiple measures of rapid response as predictors of remission in cognitive behavior therapy for bulimia nervosa. Behav Res Ther 64: 9-14.

6. McElroy SL, Guerdjikova Al, Mori N, O'Melia AM (2012) Current pharmacotherapy options for bulimia nervosa and binge eating disorder. Expert Opin Pharmacother 13: 2015-2026.

7. Zhu AJ, Walsh BT (2002) Pharmacologic treatment of eating disorders. Can J Psychiatry 47: 227-234.

8. Aigner M, Treasure J, Kaye W, Kasper S, WFSBP Task Force On Eating Disorders (2011) World Federation of Societies of Biological Psychiatry (WFSBP) guidelines for the pharmacological treatment of eating disorders. World J Biol Psychiatry 12: 400-443.

9. Tripp AC (2010) Bupropion, a brief history of seizure risk. Gen Hosp Psychiatry 32: $216-217$

10. Bacaltchuk J, Hay P (2003) Antidepressants versus placebo for people with bulimia nervosa. Cochrane Database Syst Rev 4: CD003391.
11. New International Version (2012) The Holy Bible. Proverbs 3:5-6. Zondervan, Grand Rapids, MI.

12. Yager J, Devlin MJ, Halmi KA, Herzog DB, Mitchell JE, et al. (2006) Treatment of patients with eating disorders ( $3^{\text {rd }}$ edn.). Am J Psychiatry 163: 4-54.

13. Shapiro JR, Berkman ND, Brownley KA, Sedway JA, Lohr KN, et al. (2007) Bulimia nervosa treatment: a systematic review of randomized controlled trials. Int J Eat Disord 40: 321-336.

14. Hay P, Chinn D, Forbes D, Madden S (2014) Royal Australian and New Zealand College of Psychiatrists clinical practice guidelines for the treatment of eating disorders. Aust N Z J Psychiatry 48: 977-1008.

15. Hsu LK, Clement L, Santhouse R, Ju ES (1991) Treatment of bulimia nervosa with lithium carbonate. A controlled study. J Nerv Ment Dis 179: 351-355.

16. Faris PL, Kim SW, Meller WH (2000) Effect of decreasing afferent vagal activity with ondansetron on symptoms of bulimia nervosa: a randomised, double-blind trial. Lancet 355: 792-797.

17. Broft Al, Spanos A, Corwin RL (2007) Baclofen for binge eating: an open-label trial. Int J Eat Disord 40: 687-691.

18. Naessen S, Carlstrom K, Bystrom B, Pierre Y, Hirschberg AL (2007) Effects of an antiandrogenic oral contraceptive on appetite and eating behavior in bulimic women. Psychoneuroendocrinology 32: 548-554.

19. Malhotra S, King KH, Welge JA, Brusman-Lovins L, McElroy SL (2002) Venlafaxine treatment of binge-eating disorder associated with obesity: a series of 35 patients. J Clin Psychiatry 63: 802-806.

20. Hazen E, Fava M (2010) Successful duloxetine treatment of a binge eating disorder: a case report. J Psychopharmacol 24: 1269-1272.

21. Mago R, Forero G, Greenberg WM, Gommoll C, Chen C (2013) Safety and tolerability of levomilnacipran ER in major depressive disorder: results from an open-label, 48-week extension study. Clin Drug Investig 33: 761-71.

22. (2014) Fetzima-levomilnacipran hydrochloride capsule, extended release Daily Med, U.S. National Library of Medicine. Updated July 2014.

23. Citrome $L$ (2013) Levomilnacipran for major depressive disorder: a systematic review of the efficacy and safety profile for this newly approved antidepressantwhat is the number needed to treat, number needed to harm and likelihood to be helped or harmed? Int J Clin Pract 67: 1089-1104.

24. Bakish D, Bose A, Gommoll C, Chen C, Nunez R, et al. (2014) Levomilnacipran ER $40 \mathrm{mg}$ and $80 \mathrm{mg}$ in patients with major depressive disorder: a phase III, randomized, double-blind, fixed-dose, placebo-controlled study. J Psychiatry Neurosci 39: 40-49.

25. Sambunaris A, Bose A, Gommoll CP, Chen C, Greenberg WM, et a (2014) A phase III, double-blind, placebo-controlled, flexible-dose study of levomilnacipran extended-release in patients with major depressive disorder. $\mathrm{J}$ Clin Psychopharmacol 34: 47-56.

26. Montgomery SA, Mansuy L, Ruth A, Bose A, Li H, et al. (2013) Efficacy and safety of levomilnacipran sustained release in moderate to severe major depressive disorder: a randomized, double-blind, placebo-controlled, proof-ofconcept study. J Clin Psychiatry 74: 363-369.

27. Perkins SJ, Murphy R, Schmidt U, Williams C (2006) Self-help and guided selfhelp for eating disorders. Cochrane Database Syst Rev 2006: CD004191.

28. Akrawi D, Bartrop R, Potter U, Touyz S (2015) Religiosity, spirituality in relation to disordered eating and body image concerns: A systematic review. J Eat Disord 3: 29 .

29. Richards PS, Berrett ME, Hardman RK, Eggett DL (2006) Comparative efficacy of spirituality, cognitive, and emotional support groups for treating eating disorder inpatients. Eat Disord 14: 401-415. 